\title{
Surface and Mirror Twin Grain Boundary Segregation in Nd:YAG: An Atomistic Simulation Study
}

\author{
Ulrich Aschauer ${ }^{\dagger}$ and Paul Bowen \\ Laboratoire de Technologie des Poudres, Ecole Polytechnique Fédérale de Lausanne, Lausanne, Switzerland \\ Stephen C. Parker \\ Department of Chemistry, University of Bath, Bath BA2 7AY, UK
}

\begin{abstract}
Ceramic lasers have advantages such as better optical homogeneity, cheaper production, and more freedom in shape compared with monocrystalline lasers. However, equal or better laser performances are required. Interface segregation is important as ceramics contain a high number of interfaces, segregation to which may locally alter the concentration of luminescent dopants, which in turn may influence laser performance. The present work applies atomistic simulation techniques to investigate the segregation of neodymium (Nd) dopants to surfaces and mirror twin grain boundaries in yttrium aluminum garnet (YAG). These results allow a better understanding of interfacial segregation and its influence on laser performance of Nd:YAG ceramics.
\end{abstract}

\section{Introduction}

$\mathrm{N}$ EODYMIUM-doped yttrium aluminum garnet $(\mathrm{Nd}: Y A G$, $\left.\mathrm{Nd}: \mathrm{Y}_{3} \mathrm{Al}_{5} \mathrm{O}_{12}\right)$ has become, since its discovery by Geusic et al., ${ }^{1}$ a well-known high-performance laser material. It is generally used in the form of single crystals grown by the Czochralski method. However, as this method of growth is extremely slow and as it is difficult to homogeneously dope the YAG with more than 1.4 at. $\%$ of $\mathrm{Nd}$, recent advances toward the use of polycrystalline Nd:YAG ceramics have been made, notably by Ikesue and colleagues. ${ }^{2-4}$ Significant contributions on synthesis and processing have also been made by Ueda and colleagues ${ }^{5-7}$ and Lupei and colleagues. ${ }^{8,9}$ Besides a higher optical homogeneity, the major advantages of the ceramic route would be a lower cost and higher production rate as well as much more freedom in shape - even composite structures can be produced as shown by Ikesue and Aung, ${ }^{4}$ which may lead to significant improvements in laser performance and quality.

L'huillier et al. ${ }^{10}$ have shown that it is possible to grow monocrystalline $\mathrm{Nd}$ :YAG containing 2 at. \% of $\mathrm{Nd}$, and even higher concentrations of 2.5 at. $\%$ have been reported by the use of special growth techniques such as thermal-gradient growth. ${ }^{9}$ Ceramic materials on the other hand can be doped with up to 8 at. \% of $\mathrm{Nd}$ (Konoshima Chemicals Co. Ltd., Osaka, Japan); however, the exact location of these dopants is not fully known as the resolution of measurement by fluorescence life-time as reported by L'huillier et al. ${ }^{11}$ is limited to $50 \mu \mathrm{m}$. Segregation from the bulk toward the interfaces is likely to occur due to the difference in ionic size between Nd $(0.995 \AA)$ and Y $(0.900 \AA)$, the $\mathrm{Nd}$ being substituted on $\mathrm{Y}$ sites in the YAG structure. We have previously studied segregation behavior to surfaces in

L.-Q. Chen-contributing editor

Manuscript No. 24102. Received December 14, 2007; approved April 23, 2008.

This work was supported by the European Sixth Framework Integrated Project Nanoker No. NMP3-CT-2005-515784

Author to whom correspondence should be addressed. e-mail: uli.aschauer@epfl.ch
YAG by computational techniques, ${ }^{12}$ and it was found that $\mathrm{Nd}$ segregates to YAG surfaces quite significantly, resulting in surface to bulk dopant concentration ratios of up to 1300 .

An important phenomenon occurring in Nd:YAG lasers is concentration quenching, which means that with increasing $\mathrm{Nd}$ concentration the spacing between $\mathrm{Nd}$ ions decreases and crossrelaxation between the dopant ions reduces the emission lifetime of the luminescent $\mathrm{Nd}$ ions. This effect decreases the laser power and has been measured by Deb et al., ${ }^{13}$ Merkle et al., ${ }^{14}$ and Dong et al..$^{15}$ and modeled by Huang et al. ${ }^{16}$ As there may be local variations in concentration due to segregation in polycrystalline ceramic lasers, localized concentration quenching may occur.

The laser performance of single crystals and ceramics has been compared by Lupei et al. ${ }^{8}$ and it has been found that ceramic lasers can, under carefully chosen pumping conditions, yield the same performances as single crystals. Further advances in the field of polycrystalline Nd:YAG lasers may be achieved by knowledge-based microstructure control, the target values of grain size and dopant concentration with predicted degree of segregation may be provided by simulations. In a previous publication, ${ }^{12}$ we reported our results on surface segregation that would be expected to occur in a doped Nd:YAG powder before sintering or to free surfaces during sintering. In the present publication this approach is taken a step further, where we carry out a more detailed study of surfaces as well as segregation to mirror twin grain boundaries, which can serve as a basis for the quest of optimizing Nd:YAG ceramic laser materials.

\section{Computational Method}

The computer code METADISE ${ }^{17}$ was used for the creation and subsequent energy minimization of all structures in this article. A detailed description can be found in our previous arti$\mathrm{cle}^{12}$ and only a short summary is given here, pointing out differences and additions to the previous methodology. The basic data for the simulation is the crystallographic unit cell of YAG, which was taken from the Rietveld refined neutron diffraction data recorded at $10 \mathrm{~K}$ published by Rodic et al., ${ }^{18}$ and an interatomic potential set published by Lewis and Catlow, ${ }^{19}$ previously applied with success to a large range of garnets. ${ }^{20}$ This potential describes the interatomic forces between a pair of ions by a combination of long-range coulombic forces as well as a Buckingham potential for the short-range interaction. In order to model the polarizability of the oxygen ion, a coreshell model as first described by Dick and Overhauser ${ }^{21}$ has been used.

A comparison of experimental bulk properties ${ }^{18,22-24}$ with those predicted by the potential model is given in Table I. As it can be seen, the structural parameters of the lattice are very well reproduced, whereas the mechanical and dielectric properties show higher deviations. This means that the current potentials are well suited to investigate atomic structures. However, 
Table I. Comparison of Experimental Bulk Properties of Yttrium Aluminum Garnet (YAG) with those Calculated

\begin{tabular}{lcccccc}
\hline & & \multicolumn{3}{c}{ Elastic constants } & \\
\cline { 2 - 5 } & Lattice constant & $\mathrm{C}_{11}$ & $\mathrm{C}_{12}$ & $\mathrm{C}_{44}$ & Dielectric constant \\
\cline { 2 - 6 } Property & $(\AA)$ & $(\mathrm{GPa})$ & $(\mathrm{GPa})$ & $(\mathrm{GPa})$ & $(-)$ & $(116$ \\
\hline Experiment & 11.9927 & 339 & 114 & 126.13 & 3.28 & 2.55 \\
Simulation & 11.9287 & 399.25 & 128.69 & 1.82 & 1.60 \\
\hline
\end{tabular}

The lattice constants are the ones determined by Rodic et al. ${ }^{18}$ at $10 \mathrm{~K}$, the elastic constants have been measured at $300 \mathrm{~K}$ by Stoddart et al., ${ }^{22}$ the dielectric constant is the one reported by Tomiki et al..$^{23}$ for room temperature, and the refractive index was measured by Bond ${ }^{24}$ at room temperature for a wavelength of $1 \mu \mathrm{m}$.

dielectric properties calculated based on the current potentials should be interpreted with care, the potentials should be refined, or first principle methods be applied for optical property calculations. A very important property of the employed potential set is that it describes under-coordinated environments reasonably well, without leading to unphysical relaxations. ${ }^{25}$ Many authors $^{26-30}$ have applied it to bulk, surfaces, and interfaces of different materials and found it to qualitatively reproduce the structures as well as quantitative properties such as interfacial energies, illustrating its suitability for the study of surfaces and grain boundaries.

Experimental data published by Cherepanova et $a l^{31}$ and Roberts and Elwell ${ }^{32}$ suggest that the (110) and (112) surfaces are dominant in the morphology of YAG crystals. In addition, the (100) and (111) surfaces were studied as low-index surfaces are likely to have low interfacial energies. Surfaces can be cleaved at different depths perpendicular to a direction defining the surface normal. Depending on the position of this cleaving plane, different atoms will be exposed at the surface. Some of these so-called surface terminations or cuts will present a dipole moment perpendicular to the surface, which makes them physically unstable because the applied periodic boundary conditions would result in an infinite dipole. For the four aforementioned surface planes, all surface terminations without a dipole moment were constructed resulting in five, six, seven, and three possible terminations for the four directions, respectively. In the following, the terminations will be designated by consecutive numbers-" $(111)$ cut 4" meaning for example to the fourth cut perpendicular to the [111] surface normal direction. All interfaces were described using the two-region model. This model splits the crystal into two parts, an interface region where the ions are free to relax to their equilibrium position and a bulk region where they are held fixed, as shown in Fig. 1(a). All surfaces were allowed to relax to their minimum energy configuration.

Grain boundary structures were constructed by mirroring the unrelaxed surface, which results in two interface regions as well as two bulk regions sitting back to back as shown in Fig. 1(b). A gap of $2 \AA$ was introduced between the two half-crystals by shifting the top half upwards. The top crystal was then displaced as a whole rigid block with respect to the lower one in the two dimensions of the interface plane. Steps of $0.5 \AA$ were first applied in one of the two directions until a line was finished then making a step of $0.5 \AA$ in the other direction to scan the next line. At each point the structure was allowed to relax until the energy converged, permitting a rigid displacement of the whole upper half-crystal in the (a)

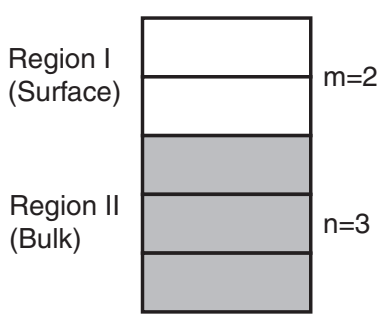

(b)

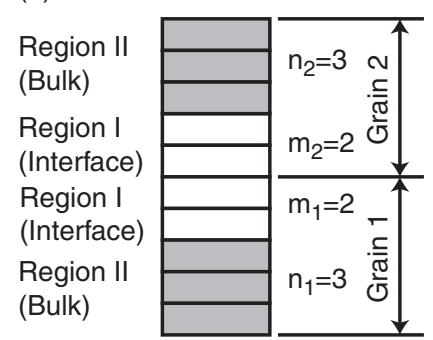

Fig. 1. The two region model for (a) surface modeling and (b) grain boundary modeling $(148 \mathrm{~mm} \times 58 \mathrm{~mm}(600 \times 600 \mathrm{DPI}))$. direction normal to the interface plane. This allows the gap introduced between the two half-crystals to either close when favorable interactions between top and bottom crystal exist, resulting in a lowering of the energy or open when like-charge repulsion dominates, which increases the energy. The relaxed energy related to the relative positioning of the two half-crystals can thus be monitored on a grid in the two dimensions of the interface plane. The grid point representing the overall minimum energy on this energy surface was assumed to be the grain boundary configuration.

It should be noted that this method of grain boundary construction introduces certain simplifications. Firstly, only mirror images of the exact same surface are joined to form a grain boundary, whereas two different surfaces or even terminations with a dipole could result in a lower energy. Secondly, grain boundary faceting is not explicitly taken into account in this method of construction. During relaxation of the structures, the position and shape of the boundary plane may change freely; however, the energy barriers associated with an important structural change such as grain boundary faceting are most likely too high to overcome. Despite these points, the present choice is seen as a reasonable decision with respect to the grain boundary types looked at as otherwise the computational effort and the complexity of the problem would be overwhelming.

In a previous study, ${ }^{12}$ the segregation was studied only on the lowest energy surface termination along each crystallographic direction. However, in this study, as the segregation calculations are computationally cheap compared with the determination of the grain boundary rigid shift, the segregation was studied to all possible surface terminations as well as their respective mirror twin boundaries.

When inserting a single dopant ion into the interface structure, the energy of this incorporation can be compared with the incorporation into the infinite and otherwise perfect bulk crystal. This change in energy represents the driving force for a dopant to migrate from the bulk to the interface, also known as the enthalpy of segregation, which is given by

$$
\begin{aligned}
\Delta H_{\text {seg }, i}(1) & =\Delta H_{\text {dopant }, i}^{\text {interface }}-\Delta H_{\text {dopant }}^{\text {bulk }} \\
& =\left(H_{\text {doped }, i}^{\text {interface }}-H_{\text {undoped }}^{\text {interface }}\right)-\Delta H_{\text {dopant }}^{\text {bulk }}
\end{aligned}
$$

In this equation, $\Delta H_{\text {dopant, } i}^{\text {interface }}$ and $\Delta H_{\text {dopant }}^{\text {bulk }}$ are the energies of incorporating a dopant on a certain site $i$ at the interface and in the bulk, respectively. The incorporation at the interface can be expressed as a function of the lattice energies $\Delta H_{\mathrm{doped}, i}^{\text {interface }}$ and $\Delta H_{\text {undoped }}^{\text {interface }}$ of the structure with and without a dopant on site $i$, respectively, which are calculated based on the interatomic potentials. The difference in enthalpy for the incorporation in the bulk $\Delta H_{\text {dopant }}^{\text {bulk }}$ is also calculated from the interatomic potentials using the Mott-Littleton approach ${ }^{33}$ implemented in the GULP $^{34}$ code, which describes dopant ions in the bulk at infinite dilution.

The next step is the insertion of multiple dopant ions into the interface structure. The number of possible different configurations when inserting up to $n$ dopant ions onto $n$ host sites in a crystal is $2^{n}$. As the number of atoms and thus possible incorporation sites increase with the interface area of the periodic cell, 
Table II. Surface and Grain Boundary Energies for the Undoped and the Doped Case

\begin{tabular}{|c|c|c|c|c|c|}
\hline \multirow[b]{2}{*}{ Surface } & \multirow[b]{2}{*}{ Cut } & \multicolumn{2}{|c|}{ Surface energy $\left(\mathrm{J} / \mathrm{m}^{2}\right)$} & \multicolumn{2}{|c|}{ Grain boundary energy $\left(\mathrm{J} / \mathrm{m}^{2}\right)$} \\
\hline & & Undoped & Doped & Undoped & Doped \\
\hline \multirow[t]{6}{*}{$(100)$} & 1 & 2.32 & 2.24 & 2.09 & 1.97 \\
\hline & 2 & 1.81 & 1.77 & 1.93 & 1.73 \\
\hline & 3 & 2.31 & 2.26 & 3.26 & 2.81 \\
\hline & 4 & 2.40 & 1.94 & 2.95 & 2.52 \\
\hline & 5 & 1.91 & 1.86 & 2.84 & 2.82 \\
\hline & Mean & 2.15 & 2.01 & 2.61 & 2.37 \\
\hline \multirow[t]{7}{*}{ (110) } & 1 & 2.43 & 2.23 & 1.41 & 1.31 \\
\hline & 2 & 2.36 & 2.33 & 1.79 & 1.61 \\
\hline & 3 & 2.75 & 2.25 & 0.82 & 0.82 \\
\hline & 4 & 2.61 & 2.49 & 2.52 & 2.52 \\
\hline & 5 & 2.67 & 2.47 & 2.31 & 2.31 \\
\hline & 6 & 3.05 & 2.28 & 1.23 & 1.10 \\
\hline & Mean & 2.65 & 2.34 & 1.68 & 1.61 \\
\hline \multirow[t]{8}{*}{ (111) } & 1 & 2.33 & 2.33 & 1.90 & 1.78 \\
\hline & 2 & 2.25 & 2.11 & 1.78 & 1.73 \\
\hline & 3 & 2.92 & 2.59 & 2.38 & 2.28 \\
\hline & 4 & 2.48 & 2.21 & 3.49 & 3.43 \\
\hline & 5 & 2.39 & 2.18 & 3.08 & 2.94 \\
\hline & 6 & 2.76 & 2.68 & 3.36 & 3.32 \\
\hline & 7 & 2.95 & 2.83 & 3.13 & 2.93 \\
\hline & Mean & 2.58 & 2.42 & 2.73 & 2.63 \\
\hline \multirow[t]{4}{*}{ (112) } & 1 & 2.36 & 2.22 & 1.65 & 1.65 \\
\hline & 2 & 2.61 & 2.52 & 2.70 & 2.54 \\
\hline & 3 & 2.50 & 2.36 & 2.86 & 2.80 \\
\hline & Mean & 2.49 & 2.37 & 2.40 & 2.33 \\
\hline
\end{tabular}

For the doped case the equilibrium concentration (as reported in Table III) at minimum interfacial energy has been considered.

$n$ can easily reach values of 30 and more for the larger (111) and (112) interfaces calculated. As relaxation of all these permutations clearly is computationally too expensive, a method not requiring the explicit relaxation of the energy for every configuration had to be found. Such a method should serve to sample all the configurations, select the ones that are likely to have a low energy and relax the energy only for those, discarding the higher energy ones. It was therefore assumed that the probability of a certain arrangement of dopant ions on host sites is given as a function of the enthalpies of segregation as shown in the following equation:

$$
p=\prod_{i}^{\text {occup. sites }} \frac{\exp \left(-\Delta H_{\mathrm{seg}, i}(1)\right)}{1+\exp \left(-\Delta H_{\mathrm{seg}, i}(1)\right)}
$$

This equation is one of the standard equations for transition probabilities in the Monte Carlo method, which is here multiplied over all occupied sites in the configuration. Also omitted is the dividing factor $k T$ usually found in this equation as the result looked for in this particular case is not a physical transition probability at a certain temperature but only an indication of the likeliness of a certain configuration on an arbitrary scale.

This allows the configurations to be sorted by descending probability and to explicitly minimize the energy uniquely for the most probable ones for each number of dopants at the interface. The method is based on the hypothesis that sites do not interact, which is not true at higher dopant concentrations. To resolve this ambiguity, it was shown that for a test set of surfaces the minimal energy structure was always within the first 10 configurations when sorted according to the above method. Therefore, the top 15 configurations for each dopant configuration in every structure were computed, reducing the computational cost from $2^{n}$ to $15 n$ calculations.

The coverage dependent enthalpy of segregation (Eq. (3)), as introduced by Mackrodt and Tasker ${ }^{35}$ is an adaption of Eq. (1) for multidopant configurations. This difference in enthalpy gives the driving force for segregation of the $n$th dopant ion from the bulk to the interface. It was evaluated by considering the lowest energies for each number of dopants $n$ as determined by the aforementioned approach.

$$
\Delta H_{\mathrm{seg}}(n)=H_{n}^{\text {interface }}-H_{n-1}^{\text {interface }}-\Delta H_{\mathrm{dopant}}^{\mathrm{bulk}}
$$

$H_{n}^{\text {interface }}$ is the calculated lattice energy of the interface structure containing $n$ dopant ions, $H_{n-1}^{\text {interface }}$ the energy with $n-1$ dopant ions, and $\Delta H_{\text {dopant }}^{\text {bulk }}$ as in Eq. (1) the change in enthalpy when incorporating a dopant ion in the bulk.

Another interesting property is the change in enthalpy associated with the dissolution of a dopant ion originating from its stable oxide into the host crystal. Considering the reaction given in Eq. (4), the enthalpy of the solution per dopant ion as a function of the number of dopant ions can be calculated as given by Eq. (5)

$$
\begin{aligned}
& \text { undoped }+n \cdot \mathrm{NdO}_{1.5} \Rightarrow \text { doped }+n \cdot \mathrm{YO}_{1.5} \\
& \Delta H_{\mathrm{sol}}(n)=\frac{H_{n}^{\text {interface }}+n H_{\mathrm{YO}_{1.5}}-H_{\text {undoped }}^{\text {interface }}-n H_{\mathrm{NdO}_{1.5}}}{n}
\end{aligned}
$$

where $H_{\mathrm{YO}_{1.5}}$ and $H_{\mathrm{NdO}_{1.5}}$ are the lattice enthalpies of the pure oxides per cation in the bulk and $H_{\text {undoped }}^{\text {interface }}$ and $H_{n}^{\text {interface }}$ as before the calculated lattice energies of the undoped and doped interface, respectively. The new interfacial energy can then be approximated by Eq. (6).

$$
\gamma_{\text {doped }}(n)=\gamma_{\text {undoped }}+\frac{n}{A} \Delta H_{\text {sol }}(n)
$$

where $\gamma_{\text {undoped }}$ is the interface energy of the undoped interface, $n$ the number of dopants, and $A$ the interface area.

\section{Results and Discussion}

\section{(1) Interfacial Energies}

We have calculated the undoped as well as the Nd-doped structure for surfaces and mirror twin grain boundaries in YAG. The respective interfacial energies are given in Table II. Firstly, these results show that the (100) surface is the most stable followed by the (111), (110), and (112) surfaces, the latter two having similar surface energies. The equilibrium morphology of such a crystal as determined by a Wulff construction is given in Fig. 2(a). This sequence of stable surfaces is retained for the doped case, the resulting equilibrium shape is given in Fig. 2(b). The surface areas change only slightly as a result of doping: (100), $66.8 \%$ $61.2 \%$; (110), $5.0 \%-6.8 \%$; and (111), $28.2 \%-32.0 \%$. It has to be noted that for the (110) surface a different cut becomes the lowest energy surface termination when doped, which would mean a shift of the surface plane. Another finding is that all surfaces can lower their surface energies by incorporating dopants. In our previous article we found the (112) surface to be the second most
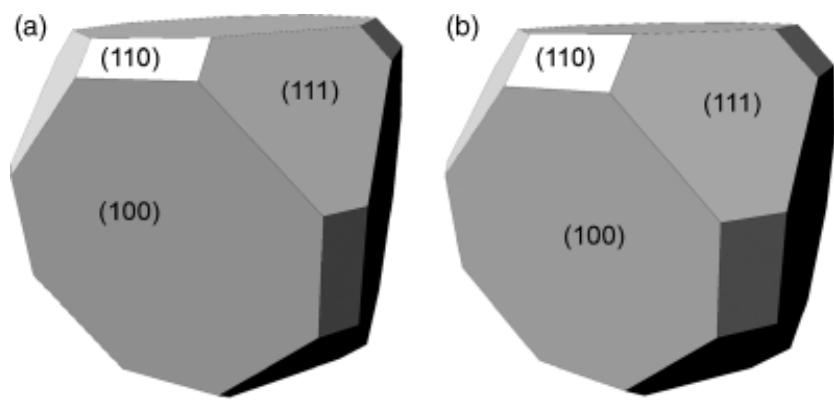

Fig. 2. Morphologies calculated from (a) the undoped surface energy and (b) the doped surface energy $(99 \mathrm{~mm} \times 47 \mathrm{~mm}(300 \times 300 \mathrm{DPI}))$. 
(a)
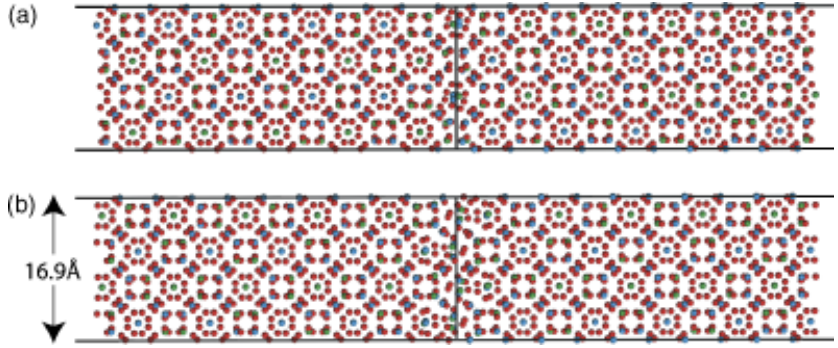

Fig. 3. (110) mirror twin grain boundary structures viewed along the [010] direction. (a) The most stable boundary (cut 3) and (b) the least stable boundary (cut 4). Neither accepts any dopants at the interface. Color code: red, oxygen; blue, aluminum; green, yttrium $(180 \mathrm{~mm} \times 85$ $\mathrm{mm}(300 \times 300 \mathrm{DPI}))$.

stable surface. During the present work it was found that in the previous work, limited by the number of atoms the code could treat in the interface region, the too-shallow interface region depth resulted in an incomplete relaxation of the surface. The previous interface structure showed a small island of oxygen atoms sticking $0.2 \AA$ out of the surface, whereas the current surface is atomically flat. In the current work, as the relaxation of the surface is less constrained, the result should be more reliable even as the surface energy increases from 1.94 to $2.36 \mathrm{~J} / \mathrm{m}^{2}$. This fact also results in the disappearance of the (112) face from the equilibrium morphology.

For the undoped grain boundaries, the (110) mirror twin boundary has by far the lowest interfacial energy, followed by the (112), (111), and (100) mirror twins. When doped, the same cuts stay stable, the sequence of interfacial energy also being conserved. However, the (100) mirror twin approaches the interfacial energy of the (111) mirror twin. It can be seen that of the most stable grain boundaries only the (100) and (111) mirror twins reduce their interfacial energy by taking up dopants. Grain boundary structures for the most and the least stable (110) mirror twins, neither of which accepts dopants, are shown in Fig. 3. Figure 4 shows the structures of the most and the least stable (112) mirror twins, where only the least stable accept dopants.

\section{(2) Enthalpies of Segregation}

The coverage-dependent values for the enthalpy of segregation are reported in Fig. 5 for the four investigated surfaces and their mirror twin boundaries. For clarity of presentation, only the most and the least stable cut of each face is shown, the rest being situated in between these two extremes. For the higher index surfaces, the concentrations investigated are lower, due to the higher surface area; however, even for the case of the (112) surface, the highest concentrations will never be attained in the experiment, as will be illustrated later during the discussion of the equilibrium interfacial energy. It can be seen that some surfaces and grain boundaries have extremely high enthalpies of segregation for the first dopant ion; however, for the second dopant ion the enthalpies of segregation lie in the same range as for the other terminations. The incorporation of the first dopant is thus extremely favorable at these interfaces. Another important result seen in Fig. 5 is the oscillatory nature of some of the curves. This indicates that the incorporation of a single dopant is less favorable than the incorporation of a pair, suggesting the formation of patterns or clusters at the interfaces. For some cases, the value of the curve becomes positive, before falling back into the negative range. This means that a certain configuration is highly unfavorable, supporting the idea of formation of patterns. This behavior indicates some sort of interaction between the dopant ions.

\section{(3) Enthalpies of Solution and Interfacial Concentration}

In Fig. 6, the enthalpy of solution per dopant ion as a function of the interfacial concentration is shown, and the resulting interfacial energies are shown in Fig. 7. For clarity, again only the (a)

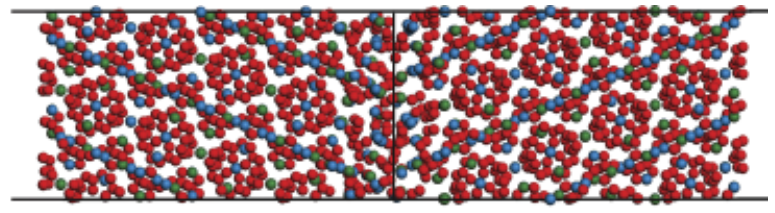

(b)
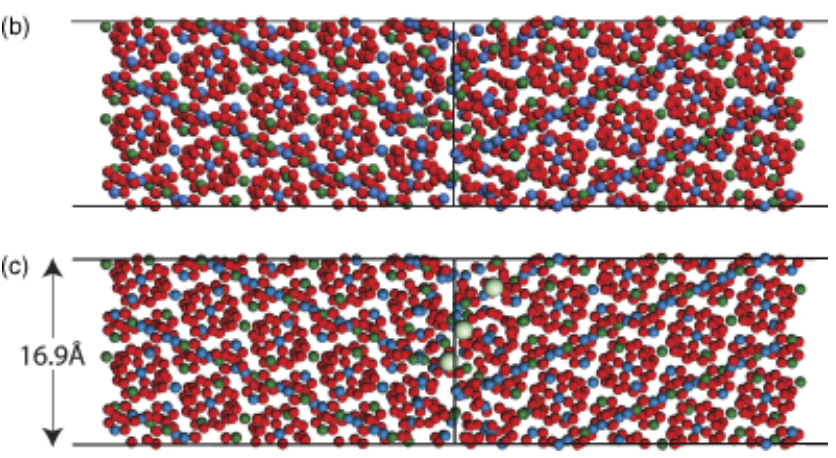

Fig. 4. (112) mirror twin grain boundary structures viewed along the [021] direction. (a) The most stable boundary (cut 1), and (b) and (c) the least stable boundary (cut 3 ) undoped and doped at its equilibrium concentration, respectively. Only the least-stable boundary accepts dopants. The dopants are shown oversized. Color code: red, oxygen; blue, aluminum; dark green, yttrium; light green, neodymium $(180 \mathrm{~mm} \times 158 \mathrm{~mm}$ $(300 \times 300 \mathrm{DPI}))$.

most and the least stable cuts are shown, the rest being in between these two extreme cases. For the [100] direction, the least stable surface and grain boundary have a high affinity for the dopants as can be seen from the very exothermic enthalpy of solution at low coverages. This results in an important lowering of the surface energies, as can be seen from the initial decrease of the curves. This effect is a lot less important for the most stable (100) surfaces and grain boundaries. However, it does occur to some extent for all cuts resulting in a minimum of interfacial energy around 3-4 dopant ions $/ \mathrm{nm}^{2}$. For the [110] direction a very exothermic solution behavior is observed only for the least stable surface, however very marked, resulting in a stabilization that brings the surface energy to a level comparable to the most stable termination for coverages of $1 \mathrm{Nd} / \mathrm{nm}^{2}$. It can be seen that the (110) grain boundaries are very stable in the undoped state, the incorporation of dopants increases the surface energy in every case, meaning that these grain boundaries should not accept any dopant ions. As seen for the enthalpy of segregation, the (111) surfaces and grain boundaries exhibit a very different behavior from the others. The surfaces and grain boundaries perpendicular to the [111] direction do not show an extreme exothermic enthalpy of solution for low coverages as all the others do. The most stable surface along this direction shows a very high capacity for dopant accommodation going up to $4 \mathrm{Nd} / \mathrm{nm}^{2}$.

The (112) surface breaks with the systematic behavior of the highest energy surfaces or grain boundaries exhibiting the extremely exothermic enthalpy of solution. For the [112] direction, it is the most stable surface and the least stable grain boundary that show this behavior. No definitive explanation for the segregation behavior of this surface could be found from the atomic structure. Eventually, the size of the surface cell could have an influence as increasing the size reduces dopant-dopant interactions, which will be present even for low coverages for the smaller surface cells. The most stable (112) grain boundary, like the (110) boundary, does not accept any dopants at all. This illustrates that extremely low-energy boundaries, which have a very regular atomic structure do not easily accommodate dopants, which would lead to a disruption of this regularity and thus an increase in energy. Despite these irregularities, it can be stated that generally low-energy interfaces show limited or even no trend toward segregation.

Because the enthalpy of dissolution does not take into account the energy of the bulk/interface system, but the one associated with the exchange between the pure dopant oxide and 

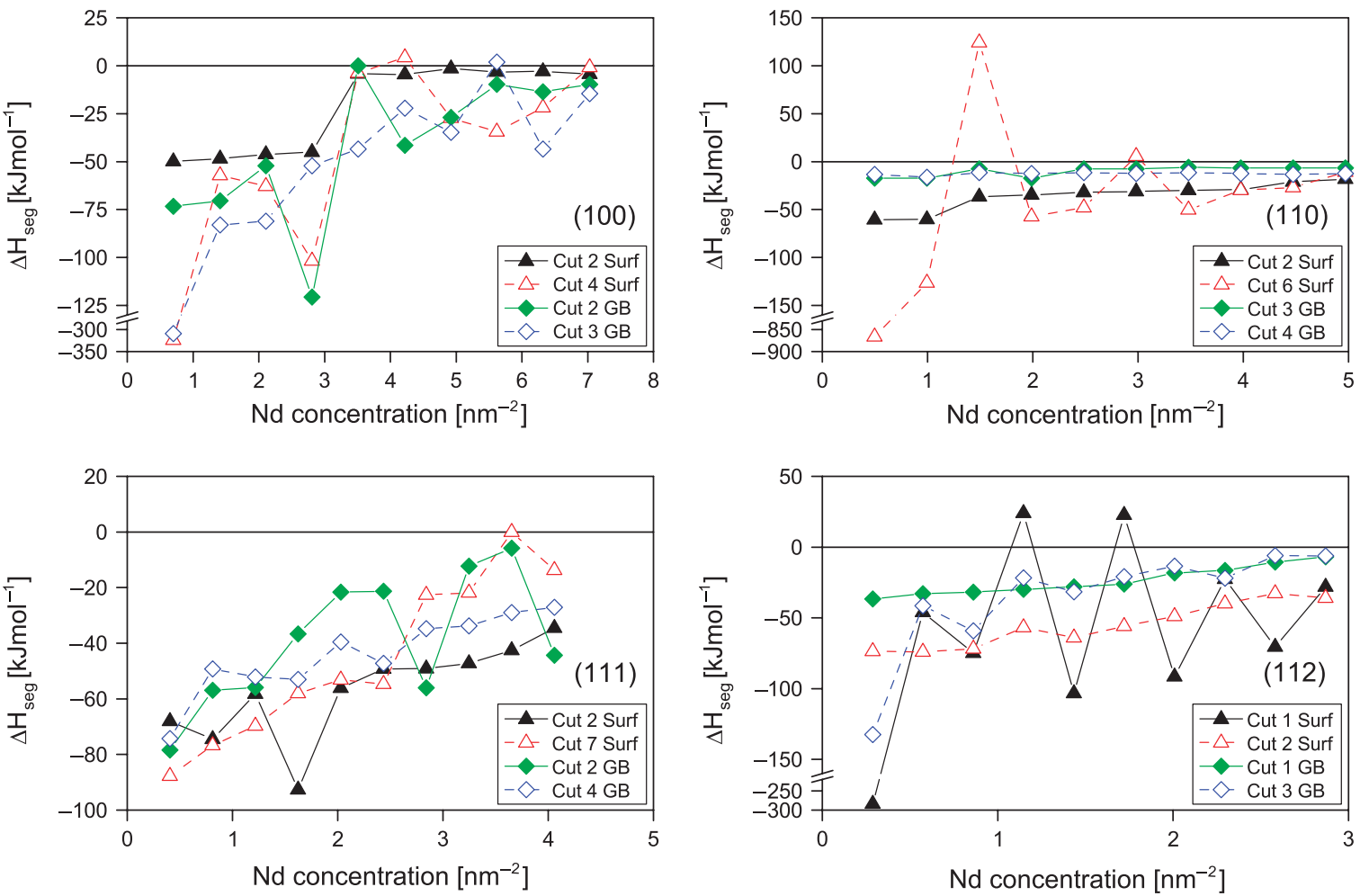

Fig. 5. Coverage-dependent enthalpy of segregation of the four surfaces (different scales and breaks are used on different graphs). For clarity only the lowest and highest energy cut of each surface end grain boundary is shown, the rest being in between these two extremes. Surfaces have triangular markers whereas grain boundaries have rhombic ones. Low-energy cuts are drawn with solid lines, whereas high-energy ones are dashed (174 mm $\times 113$ $\mathrm{mm}(600 \times 600 \mathrm{DPI}))$; Nd, neodymium.

the interface, it is reasonable to assume that segregation is governed by the minimization of the interfacial energy, which will also minimize the one of the bulk/interface system. When the dopant concentration in the system is sufficient to provide do- pant ions to all interfaces, it would be expected that the surfaces and grain boundaries show a dopant concentration equal to that of the minimum on the interfacial energy curve. At a lower dopant concentration in the system, the interfacial concentration
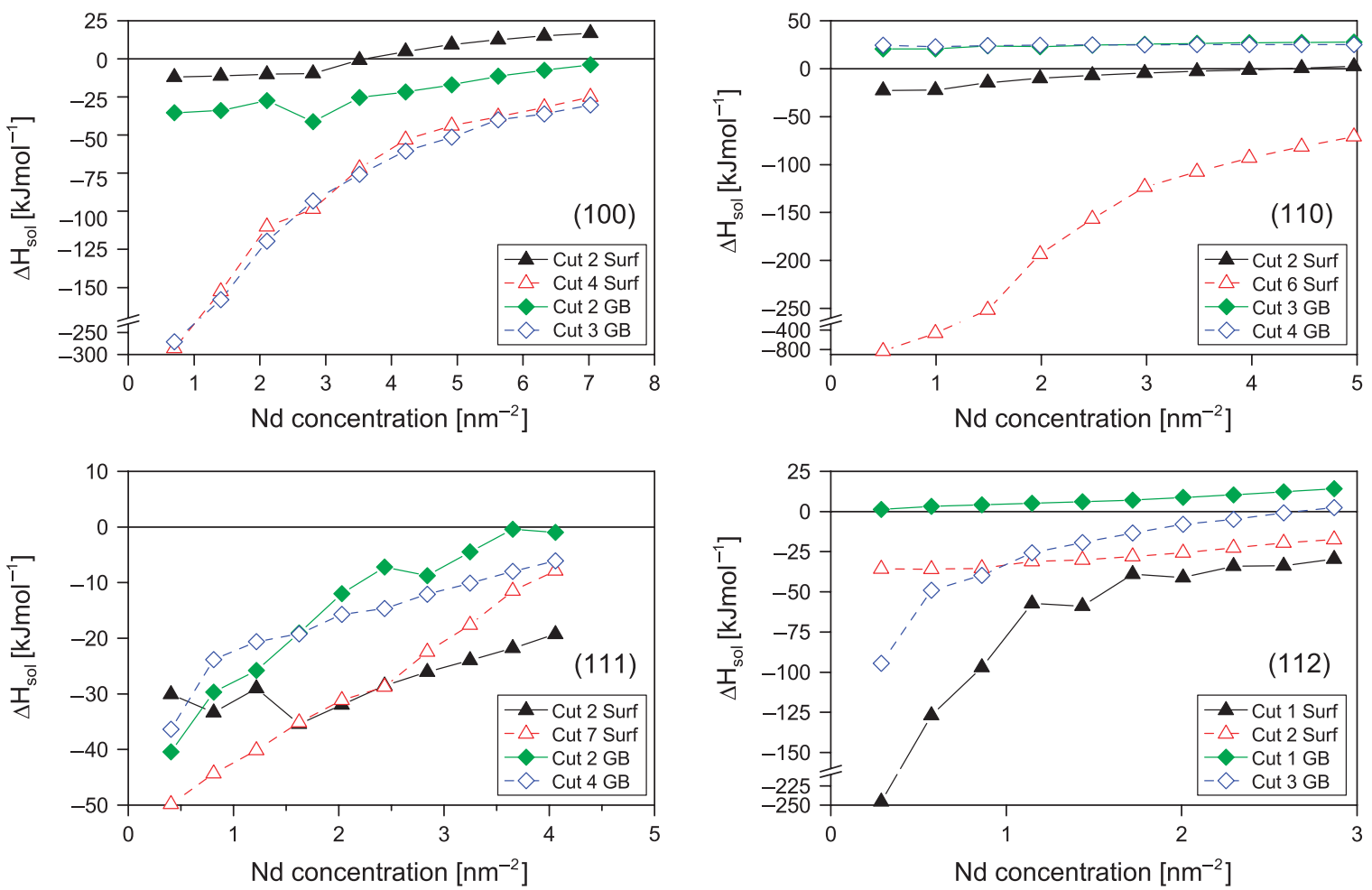

Fig. 6. Coverage-dependent enthalpy of solution per dopant ion (different scales and breaks are used on different graphs). For clarity only the lowest and highest energy cut of each surface end grain boundary is shown, the rest being in between these two extremes. Surfaces have triangular markers whereas grain boundaries have rhombic ones. Low-energy cuts are drawn with solid lines, whereas high-energy ones are dashed $(174 \mathrm{~mm} \times 112 \mathrm{~mm}$ $(600 \times 600 \mathrm{DPI})) ; \mathrm{Nd}$, neodymium. 

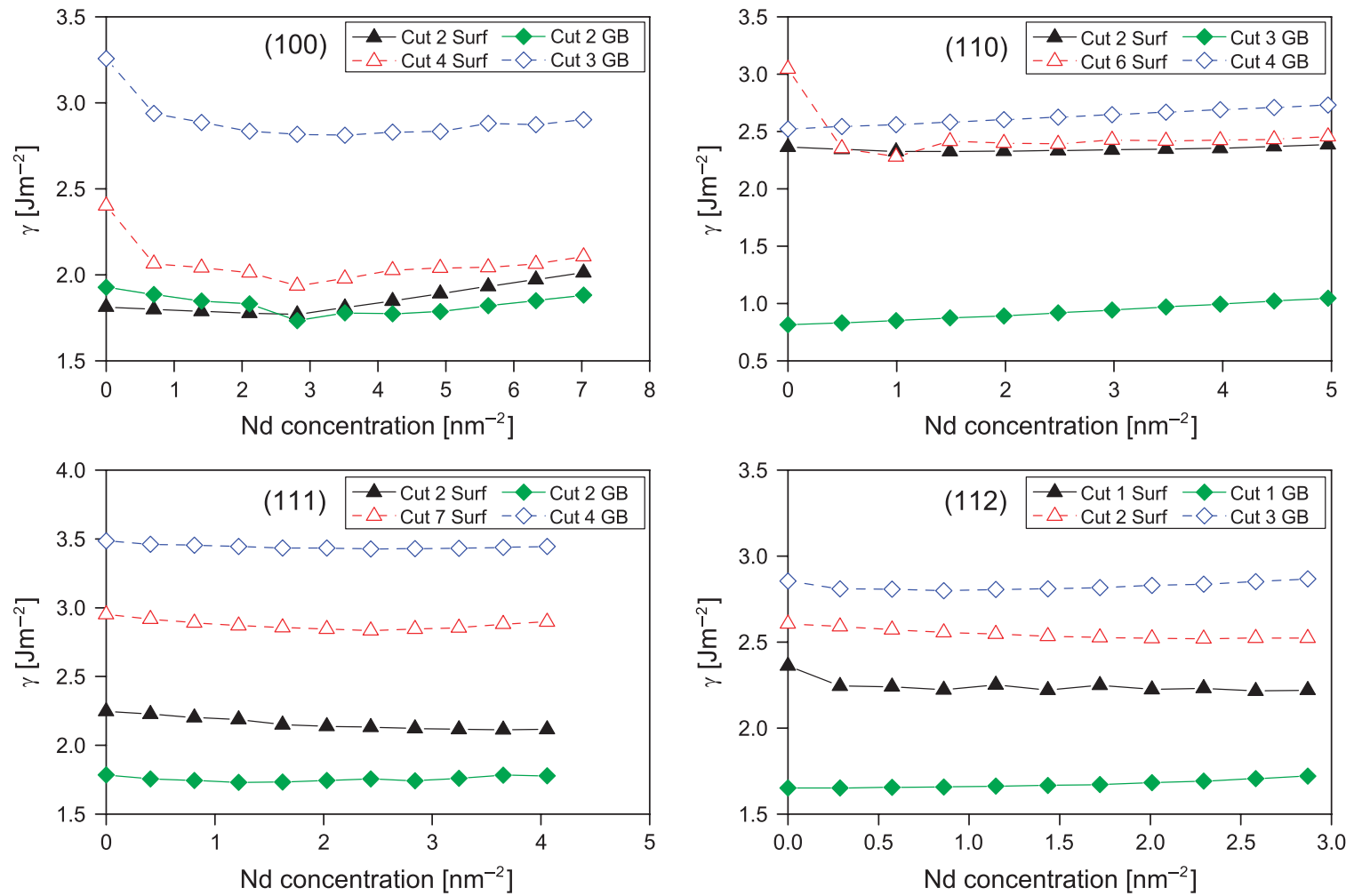

Fig. 7. Coverage-dependent interfacial energy (different scales are used on different graphs). For clarity only the lowest and highest energy cut of each surface end grain boundary is shown, the rest being in between these two extremes. Surfaces have triangular markers whereas grain boundaries have rhombic ones. Low-energy cuts are drawn with solid lines, whereas high-energy ones are dashed $(173 \mathrm{~mm} \times 113 \mathrm{~mm}(600 \times 600 \mathrm{DPI}))$; Nd, neodymium.

will be lower and the minimization of the system's energy incomplete. It can be seen that in every case this equilibrium concentration is well below the maximum concentration calculated, showing that even for the large surfaces the calculated range of dopant concentration is sufficient. For these equilibrium dopant concentrations, the atomic structure has been analyzed and characteristics of the enriched zone extracted, which can be found in Table III. It can be seen that the enriched depth varies from 0 to $5 \AA$ depending on the surface and the cut. All surfaces are covered to a certain degree, the fraction of yttrium sites occupied by the dopant ranging from 0.15 to a complete coverage of 1.0 in the enriched zone. There are some grain boundaries that do not accept any dopant ions and thus have a coverage of 0.0 , others again accept full coverage in the enriched zone. Attempts at correlating the density of the enriched zone and the interfacial energy with the dopant accommodation capacity

Table III. Characteristics of the Enriched Zone for All Surfaces and Grain Boundaries at their Lowest Interfacial Energy (Equilibrium Dopant Concentration)

\begin{tabular}{|c|c|c|c|c|c|c|c|}
\hline \multirow[b]{2}{*}{ Surface } & \multirow[b]{2}{*}{ Cut } & \multicolumn{3}{|c|}{ Surface } & \multicolumn{3}{|c|}{ Grain boundary } \\
\hline & & $\mathrm{Nd}$ conc. $\left(\mathrm{nm}^{-2}\right)$ & Depth (nm) & Coverage (-) & $\mathrm{Nd}$ conc. $\left(\mathrm{nm}^{-2}\right)$ & Depth (nm) & Coverage (-) \\
\hline \multirow[t]{5}{*}{ (100) $1.42 \mathrm{~nm}^{2}$} & 1 & 2.11 & 0.30 & 0.375 & 4.93 & $0.39 / 0.12$ & 0.500 \\
\hline & 2 & 2.82 & 0.13 & 1.000 & 2.82 & $0.23 / 0.28$ & 0.400 \\
\hline & 3 & 3.52 & 0.22 & 0.833 & 3.52 & $0.57 / 0.36$ & 0.250 \\
\hline & 4 & 2.82 & 0.43 & 0.571 & 5.63 & $0.52 / 0.41$ & 0.467 \\
\hline & 5 & 2.11 & 0.14 & 0.600 & 1.41 & $0.14 / 0.00$ & 1.000 \\
\hline \multirow[t]{6}{*}{ (110) $2.01 \mathrm{~nm}^{2}$} & 1 & 2.49 & 0.22 & 0.417 & 1.99 & $0.26 / 0.20$ & 0.200 \\
\hline & 2 & 1.00 & 0.12 & 0.333 & 2.99 & $0.18 / 0.14$ & 0.375 \\
\hline & 3 & 2.49 & 0.31 & 0.500 & 0.00 & $0.00 / 0.00$ & 0.000 \\
\hline & 4 & 1.00 & 0.11 & 0.600 & 0.00 & $0.00 / 0.00$ & 0.000 \\
\hline & 5 & 1.99 & 0.12 & 1.000 & 0.50 & $0.00 / 0.13$ & 0.250 \\
\hline & 6 & 1.00 & 0.26 & 0.167 & 0.50 & $0.00 / 0.19$ & 0.125 \\
\hline \multirow[t]{7}{*}{ (111) $2.46 \mathrm{~nm}^{2}$} & 1 & 1.22 & 0.01 & 0.500 & 4.07 & $0.32 / 0.21$ & 0.417 \\
\hline & 2 & 3.66 & 0.49 & 0.474 & 1.22 & $0.16 / 0.14$ & 0.188 \\
\hline & 3 & 2.03 & 0.41 & 0.278 & 2.44 & $0.30 / 0.11$ & 0.350 \\
\hline & 4 & 2.85 & 0.41 & 0.389 & 2.44 & $0.30 / 0.23$ & 0.273 \\
\hline & 5 & 1.22 & 0.51 & 0.150 & 4.07 & $0.36 / 0.33$ & 0.370 \\
\hline & 6 & 2.03 & 0.14 & 0.500 & 2.03 & $0.21 / 0.14$ & 0.278 \\
\hline & 7 & 2.44 & 0.15 & 0.545 & 3.66 & $0.22 / 0.17$ & 0.409 \\
\hline \multirow[t]{3}{*}{ (112) $3.49 \mathrm{~nm}^{2}$} & 1 & 2.58 & 3.28 & 0.409 & 0.00 & $0.00 / 0.00$ & 0.000 \\
\hline & 2 & 2.29 & 0.20 & 0.500 & 0.86 & $0.25 / 0.24$ & 0.094 \\
\hline & 3 & 2.87 & 0.45 & 0.400 & 0.86 & $0.04 / 0.28$ & 0.167 \\
\hline
\end{tabular}


have not shown any clear trends as the scattering of values is too large. It can, however, be stated that it does not depend solely on these two factors but also on the bonding environment, which plays a critical role in determining the equilibrium concentration of $\mathrm{Nd}$ in YAG grain boundaries.

\section{(4) Estimated Influence on Lasing Properties}

The quenching effect due to segregation can be estimated by the following method. Dong et al. ${ }^{15}$ have found a reduction of $49 \%$ in laser performance at 3 at. \% Nd in single crystals due to concentration quenching. Considering a homogeneous dopant distribution in a perfect crystal, the nearest neighbor spacing at 3 at. $\% \mathrm{Nd}$ can be calculated to be $1.3 \mathrm{~nm}$. In the simulated surfaces the nearest neighbor spacing lies between 0.2 and $0.75 \mathrm{~nm}$ and between 0.39 and $0.73 \mathrm{~nm}$ for the grain boundaries, which corresponds to a local doping level of 18 at.\% $\mathrm{Nd}$ and above. These values suggest a quenching significantly higher than the $49 \%$ reported by Dong for 3\% Nd in single crystals. Therefore, the assumption can be made that much of the dopant ions segregated at interfaces will not contribute to the lasing effect.

With the data presented here only the estimations of segregation to isolated grain boundaries is possible. The interfacial energy and concentration data will however enable the construction of microstructural models, allowing the investigation of segregation in microstructures containing a population of interfaces and as a function of grain size. One problem that needs to be addressed is to estimate how representative special grain boundaries are for the ones found in real ceramic microstructures. Mirror twin boundaries are an easy to study model system in experimental bicrystal studies as well as simulations, approaching general grain boundaries as the interfacial area of the periodic cell, and thus their $\Sigma$ value increases. However, even the larger (111) and (112) grain boundaries investigated here can probably not be considered as close to general. The relatively high number of calculated grain boundaries showing a large dispersion in interfacial energies should result in a certain degree generality when they all appear in the interface population of the microstructural model.

Another aspect that will need to be investigated is the effect of codoping with silicon, which is very often used as a sintering agent during experimental production of YAG ceramics. The tetravalent silicon ion will introduce charge-compensating defects in the structure, which if silicon is also preferentially found at interfaces could considerably influence the incorporation of $\mathrm{Nd}$ dopant ions at the interfaces.

\section{Conclusions}

In the present paper, previous calculations on Nd:YAG surface segregation have been taken a step further, on one hand looking at more surface terminations for a particular crystallographic direction as well as applying the same approach to grain boundaries.

The (100) surface was found to have the lowest surface energy, followed by the (111), (110), and (112) surfaces for both doped and undoped cases. For the grain boundaries, the sequence of increasing interfacial energy is different, with the (110) mirror twin having an extremely low-interfacial energy followed by the (112), (111), and (100) mirror twin boundaries. It is shown that generally interfaces with a high interfacial energy show a higher trend for segregation. However, the (112) mirror twin boundary does not completely comply with this rule. Also, an open and less-dense atomic structure does not seem to be the only factor governing dopant incorporation at the surfaces. The bonding environment and thus the atomic structure are likely to play a crucial role as well, which needs further investigation using ab initio methods. It is shown that interfacial doping concentrations due to segregation are high enough to result in considerable local concentration quenching. Because of this fact, not all the segregated dopants are expected to contribute to the lasing effect.
The data developed will enable the construction of microstructural models allowing the study of segregation within a microstructure of variable grain size. This computational approach should ultimately lead to knowledge-based microstructure design.

\section{References}

${ }^{1}$ J. E. Geusic, H. M. Marcos, and L. G. Vanuitert, "Laser Oscillations in NdDoped Yttrium Aluminum, Yttrium Gallium and Gadolinium Garnets," Appl. Phys. Lett., 4 [10] 182-4 (1964).

${ }^{2}$ A. Ikesue, K. Kamata, and K. Yoshida, "Synthesis of $\mathrm{Nd}^{3+}, \mathrm{Cr}^{3+}$-Codoped YAG Ceramics for High-Efficiency Solid-State Lasers," J. Am. Ceram. Soc., 78 [9] 2545-7 (1995).

${ }^{3}$ A. Ikesue, K. Kamata, and K. Yoshida, "Effects of Neodymium Concentration on Optical Characteristics of Polycrystalline Nd:YAG Laser Materials," J. Am. Ceram. Soc., 79 [7] 1921-6 (1996).

${ }^{4}$ A. Ikesue and Y. L. Aung, "Synthesis and Performance of Advanced Ceramic Lasers," J. Am. Ceram. Soc., 89 [6] 1936-44 (2006).

${ }^{5}$ J. Lu, M. Prabhu, J. Song, C. Li, J. Xu, K. Ueda, A. A. Kaminskii, H. Yagi, and T. Yanagitani, "Optical Properties and Highly Efficient Laser Oscillation of Nd:YAG Ceramics," Appl. Phys. B-Lasers Opt., 71 [4] 469-73 (2000).

${ }^{6}$ J. R. Lu, K. Ueda, H. Yagi, T. Yanagitani, Y. Akiyama, and A. A. Kaminskii, "Neodymium Doped Yttrium Aluminum Garnet $\left(\mathrm{Y}_{3} \mathrm{Al}_{5} \mathrm{O}_{12}\right)$ Nanocrystalline Ceramics - A New Generation of Solid State Laser and Optical Materials," J. Alloys Compd., 341 [1-2] 220-5 (2002).

${ }^{7}$ J. Lu, H. Yagi, K. Takaichi, T. Uematsu, J. F. Bisson, Y. Feng, A. Shirakawa, K. I. Ueda, T. Yanagitani, and A. A. Kaminskii, "110 W Ceramic $\mathrm{Nd}^{3}: \mathrm{Y}_{3} \mathrm{Al}_{5} \mathrm{O}_{12}$ Laser," Appl. Phys. B-Lasers Opt., 79 [1] 25-8 (2004).

${ }^{8}$ V. Lupei, A. Lupei, N. Pavel, T. Taira, and A. Ikesue, "Comparative Investigation of Spectroscopic and Laser Emission Characteristics under Direct 885-nm Pump of Concentrated Nd:YAG Ceramics and Crystals," Appl. Phys. B - Lasers Opt., 73 [7] 757-62 (2001).

${ }^{9}$ N. Pavel, V. Lupei, J. Saikawa, T. Taira, and H. Kan, "Neodymium Concentration Dependence of 0.94-, 1.06- and 1.34-mm Laser Emission and of Heating Effects under 809- and 885-nm Diode Laser Pumping of Nd:YAG," Appl. Phys. $B$-Lasers Opt., 82 [4] 599-605 (2006).

${ }^{10}$ J. A. L'huillier, G. Bitz, V. Wesemann, P. von Loewis, R. Wallenstein, A. Borsutzky, L. Ackermann, K. Dupre, D. Rytz, and S. Vernay, "Characterization and Laser Performance of a New Material: 2 at.\% Nd:YAG Grown by the Czochralski Method," Appl. Opt., 41 [21] 4377-84 (2002).

${ }^{11}$ J. A. L'huillier, G. Bitz, V. Wesemann, A. Borsutzky, and R. Wallenstein, "A Sensitive Non-Destructive Method for Measuring the Nd Doping Concentration in Nd:YAG with High Spatial Resolution," Appl. Phys. B-Lasers Opt., 77 [6-7] 601-6 (2003).

${ }^{12}$ U. Aschauer, P. Bowen, and S. C. Parker, "Atomistic Modeling Study of Surface Segregation in Nd:YAG," J. Am. Ceram. Soc., 89 [12] 3812-6 (2006).

${ }^{13}$ K. K. Deb, R. G. Buser, and J. Paul, "Decay Kinetics of ${ }^{4} F_{3 / 2}$ Fluorescence of $\mathrm{Nd}^{+3}$ in YAG at Room Temperature," Appl. Opt., 20 [7] 1203-6 (1981).

${ }^{14}$ L. D. Merkle, M. Dubinskii, K. L. Schepler, and S. M. Hegde, "Concentration Quenching in Fine-Grained Ceramic Nd:YAG," Opt. Exp., 14 [9] 3893-905 (2006).

${ }^{15}$ J. Dong, A. Rapaport, M. Bass, F. Szipocs, and K. Ueda, "TemperatureDependent Stimulated Emission Cross Section and Concentration Quenching in Highly Doped $\mathrm{Nd}^{3+}$ :YAG Crystals," Physica Status Solidi A-Appl. Mater. Sci., 202 [13] 2565-73 (2005).

${ }^{16}$ Z. Y. Huang, Y. D. Huang, Y. J. Chen, and Z. D. Luo, "Theoretical Study on the Laser Performances of $\mathrm{Nd}^{3}$ : YAG and $\mathrm{Nd}^{3+}: \mathrm{YVO}_{4}$ under Indirect and Direct Pumping," J. Opt. Soc. Am. B-Opt. Phys., 22 [12] 2564-9 (2005).

${ }^{17}$ G. W. Watson, E. T. Kelsey, N. H. deLeeuw, D. J. Harris, and S. C. Parker, "Atomistic Simulation of Dislocations, Surfaces and Interfaces in MgO," J. Chem. Soc.-Faraday Trans., 92 [3] 433-8 (1996).

${ }^{18}$ D. Rodic, M. Mitric, R. Tellgren, and H. Rundlof, "The Cation Distribution and Magnetic Structure of $\mathrm{Y}_{3} \mathrm{Fe}_{(5-x)} \mathrm{Al}_{x} \mathrm{O}_{12}$, , J. Magnetism Magnetic Mater., 232 [1-2] 1-8 (2001).

${ }^{19}$ G. V. Lewis and C. R. A. Catlow, "Potential Models for Ionic Oxides," J. Phys. C-Solid State Phys., 18 [6] 1149-61 (1985).

${ }^{20}$ G. A. Saunders, S. C. Parker, N. Benbattouche, and H. L. Alberts, "Elastic and Nonlinear Acoustic Properties of the Terbium Iron Garnet $\mathrm{Tb}_{3} \mathrm{Fe}_{5} \mathrm{O}_{12}$ in $\mathrm{Re}$ lation to those of Other Garnets," Phys. Rev. B, 46 [14] 8756-67 (1992).

${ }^{21}$ B. G. Dick and A. W. Overhauser, "Theory of the Dielectric Constants of Alkali Halide Crystals," Phys. Rev., 112 [1] 90-103 (1958).

${ }^{22}$ P. R. Stoddart, P. E. Ngoepe, P. M. Mjwara, J. D. Comins, and G. A. Saunders, "High-Temperature Elastic-Constants of Yttrium-Aluminum-Garnet," J. Appl. Phys., 73 [11] 7298-301 (1993).

${ }^{23}$ T. Tomiki, Y. Ganaha, T. Shikenbaru, T. Futemma, M. Yuri, Y. Aiura H. Fukutani, H. Kato, J. Tamashiro, T. Miyahara, and A. Yonesu, "OpticalSpectra of $\mathrm{Y}_{3} \mathrm{Al}_{5} \mathrm{O}_{12}$ (YAG) Single-Crystals in the Vacuum Ultraviolet Region. II," J. Phys. Soc. Japan, 62 [4] 1388-400 (1993)

${ }^{24} \mathrm{~W}$. L. Bond, "Measurement of Refractive Indices of Several Crystals," J. Appl. Phys., 36 [5] 1674 (1965).

${ }^{25}$ M. J. Davies, P. R. Kenway, P. J. Lawrence, S. C. Parker, W. C. Mackrodt, and P. W. Tasker, "Impurity Segregation to the Surfaces of Corundum-Structured Oxides," J. Chem. Soc.-Faraday Trans. 2, 85 [5] 555-63 (1989).

${ }^{26}$ O. Robach, G. Renaud, and A. Barbier, "Very-High-Quality $\mathrm{MgO}(001)$ Surfaces: Roughness, Rumpling and Relaxation," Surf. Sci., 401 [2] 227-35 (1998). 
${ }^{27}$ D. J. Harris, J. H. Harding, and S. C. Parker, "Simulations of Surfaces and Interfaces in MgO," Rad. Eff. Def. Solids, 151 [1-4] 299-304 (1999).

${ }^{28}$ C. M. Fang, S. C. Parker, and G. de With, "Atomistic Simulation of the Surface Energy of Spinel $\mathrm{MgAl}_{2} \mathrm{O}_{4}, "$ J. Am. Ceram. Soc., 83 [8] 2082-4 (2000).

${ }^{29}$ S. Kerisit, D. J. Cooke, A. Marmier, and S. C. Parker, "Atomistic Simulation of Charged Iron Oxyhydroxide Surfaces in Contact with Aqueous Solution," Chem. Commun., [24] 3027-9 (2005).

${ }^{30}$ M. Y. Lavrentiev, N. L. Allan, J. H. Harding, D. J. Harris, and J. A. Purton, "Atomistic Simulations of Surface Diffusion and Segregation in Ceramics," Comput. Mater. Sci., 36 [1-2] 54-9 (2006).
${ }^{31}$ T. A. Cherepanova, P. Bennema, Y. A. Yanson, and L. J. P. Vogels, "Morphology of Synthetic and Natural Garnets-Theory and Observations," J. Cryst. Growth, 121 [1-2] 17-32 (1992)

${ }^{32}$ K. J. Roberts and D. Elwell, "Crystal Habit and Surface-Morphology of FluxGrown Yttrium Aluminum Garnet,” J. Cryst. Growth, 53 [2] 249-60 (1981).

${ }^{33}$ M. F. Mott and M. J. Littleton, "Conduction in Polar Crystals. I. Electrolytic Conduction in Solid Salts," Trans. Faraday Soc., 34 [1] 485-99 (1938).

${ }^{34}$ J. D. Gale and A. L. Rohl, "The General Utility Lattice Program (GULP)," Mol. Simulation, 29 [5] 291-341 (2003).

${ }^{35}$ W. C. Mackrodt and P. W. Tasker, "Segregation Isotherms at the Surfaces of Oxides," J. Am. Ceram. Soc., 72 [9] 1576-83 (1989). 